\title{
MEAN LIPSCHITZ SPACES AND A GENERALIZED HILBERT OPERATOR
}

\author{
NOEL MERCHÁN
}

\begin{abstract}
If $\mu$ is a positive Borel measure on the interval $[0,1)$ we let $\mathcal{H}_{\mu}$ be the Hankel matrix $\mathcal{H}_{\mu}=\left(\mu_{n, k}\right)_{n, k \geq 0}$ with entries $\mu_{n, k}=\mu_{n+k}$, where, for $n=0,1,2, \ldots, \mu_{n}$ denotes the moment of order $n$ of $\mu$. This matrix induces formally the operator

$$
\mathcal{H}_{\mu}(f)(z)=\sum_{n=0}^{\infty}\left(\sum_{k=0}^{\infty} \mu_{n, k} a_{k}\right) z^{n}
$$

on the space of all analytic functions $f(z)=\sum_{k=0}^{\infty} a_{k} z^{k}$, in the unit disc $\mathbb{D}$. This is a natural generalization of the classical Hilbert operator. In this paper we study the action of the operators $\mathcal{H}_{\mu}$ on mean Lipschitz spaces of analytic functions.
\end{abstract}

\section{INTRODUCTION AND MAIN RESULTS}

Let $\mathbb{D}$ be the unit disc in the complex plane $\mathbb{C}$, and let $\mathcal{H o l}(\mathbb{D})$ denote the space of all analytic functions in $\mathbb{D}$. For $0<r<1$ and $f \in \mathcal{H}$ ol $(\mathbb{D})$, we set

$$
\begin{gathered}
M_{p}(r, f)=\left(\frac{1}{2 \pi} \int_{-\pi}^{\pi}\left|f\left(r e^{i \theta}\right)\right|^{p} d \theta\right)^{1 / p}, \quad 0<p<\infty, \\
M_{\infty}(r, f)=\max _{|z|=r}|f(z)| .
\end{gathered}
$$

For $0<p \leq \infty$ the Hardy space $H^{p}$ consists of those functions $f$, analytic in $\mathbb{D}$, for which

$$
\|f\|_{H^{p}}=\sup _{0<r<1} M_{p}(r, g)<\infty .
$$

We refer to [9] for the theory of Hardy spaces.

The space $B M O A$ consists of those functions $f \in H^{1}$ whose boundary values have bounded mean oscillation on $\partial \mathbb{D}$. The Bloch space $\mathcal{B}$ consists of all analytic functions $f$ in $\mathbb{D}$ with bounded invariant derivative:

$$
f \in \mathcal{B} \Leftrightarrow\|f\|_{\mathcal{B}} \stackrel{\text { def }}{=}|f(0)|+\sup _{z \in \mathbb{D}}\left(1-|z|^{2}\right)\left|f^{\prime}(z)\right|<\infty .
$$

We mention [1, 13, 23] as excellent references for these spaces. Let us recall that $B M O A \subsetneq \mathcal{B}$.

If $\mu$ is a finite positive Borel measure on $[0,1)$ and $n=0,1,2, \ldots$, we let $\mu_{n}$ denote the moment of order $n$ of $\mu$, that is, $\mu_{n}=\int_{[0,1)} t^{n} d \mu(t)$, and we let $\mathcal{H}_{\mu}$ be the Hankel matrix $\left(\mu_{n, k}\right)_{n, k \geq 0}$ with entries $\mu_{n, k}=\mu_{n+k}$. The matrix $\mathcal{H}_{\mu}$ induces formally an operator, also

2010 Mathematics Subject Classification. Primary 30H10; Secondary 47B35.

Key words and phrases. Hankel matrix, Generalized Hilbert operator, Mean Lipschitz spaces, Carleson measures.

This research is supported in part by a grant from "El Ministerio de Economía y Competitividad", Spain (MTM2014-52865-P) and by a grant from la Junta de Andalucía FQM-210. The author is also supported by a grant from "El Ministerio de de Educación, Cultura y Deporte", Spain (FPU2013/01478). 
denoted $\mathcal{H}_{\mu}$, on spaces of analytic functions in the following way: if $f(z)=\sum_{k=0}^{\infty} a_{k} z^{k} \in$ $\mathcal{H o l}(\mathbb{D})$ we define

$$
\mathcal{H}_{\mu}(f)(z)=\sum_{n=0}^{\infty}\left(\sum_{k=0}^{\infty} \mu_{n, k} a_{k}\right) z^{n}
$$

whenever the right hand side makes sense and defines an analytic function in $\mathbb{D}$.

If $\mu$ is the Lebesgue measure on $[0,1)$ the matrix $\mathcal{H}_{\mu}$ reduces to the classical Hilbert matrix $\mathcal{H}=\left((n+k+1)^{-1}\right)_{n, k \geq 0}$, which induces the classical Hilbert operator $\mathcal{H}$. The Hilbert operator is known to be well defined on $H^{1}$ and bounded from $H^{p}$ into itself, if $1<p<\infty$, but not if $p=1$ or $p=\infty$ [8].

The question of describing the measures $\mu$ for which the operator $\mathcal{H}_{\mu}$ is well defined and bounded on distinct spaces of analytic functions has been studied in a good number of papers (see [2, 7, 10, 15, 16, 19, 20, 21]). The measures in question are Carleson-type measures.

If $I \subset \partial \mathbb{D}$ is an interval, $|I|$ will denote the length of $I$. The Carleson square $S(I)$ is defined as $S(I)=\left\{r e^{i t}: e^{i t} \in I, \quad 1-\frac{|I|}{2 \pi} \leq r<1\right\}$.

If $s>0$ and $\mu$ is a positive Borel measure on $\mathbb{D}$, we shall say that $\mu$ is an $s$-Carleson measure if there exists a positive constant $C$ such that

$$
\mu(S(I)) \leq C|I|^{s}, \quad \text { for any interval } I \subset \partial \mathbb{D} .
$$

A 1-Carleson measure will be simply called a Carleson measure.

If $\mu$ is a positive Borel measure on $\mathbb{D}, 0 \leq \alpha<\infty$, and $0<s<\infty$ we say that $\mu$ is an $\alpha$-logarithmic $s$-Carleson measure [22] if there exists a positive constant $C$ such that

$$
\frac{\mu(S(I))\left(\log \frac{2 \pi}{|I|}\right)^{\alpha}}{|I|^{s}} \leq C, \quad \text { for any interval } I \subset \partial \mathbb{D} \text {. }
$$

A positive Borel measure $\mu$ on $[0,1)$ can be seen as a Borel measure on $\mathbb{D}$ by identifying it with the measure $\tilde{\mu}$ defined by

$$
\tilde{\mu}(A)=\mu(A \cap[0,1)), \quad \text { for any Borel subset } A \text { of } \mathbb{D} \text {. }
$$

In this way a positive Borel measure $\mu$ on $[0,1)$ is an $s$-Carleson measure if and only if there exists a positive constant $C$ such that

$$
\mu([t, 1)) \leq C(1-t)^{s}, \quad 0 \leq t<1 .
$$

We have a similar statement for $\alpha$-logarithmic $s$-Carleson measures.

Widom [21, Theorem 3.1] (see also [20, Theorem 3] and [19, p. 42, Theorem 7.2]) proved that $\mathcal{H}_{\mu}$ is a bounded operator from $H^{2}$ into itself if and only $\mu$ is a Carleson measure. Galanopoulos and Peláez [10] studied the operators $\mathcal{H}_{\mu}$ acting on $H^{1}$. The action of $\mathcal{H}_{\mu}$ on the Hardy spaces $H^{p}, 0<p \leq \infty$, has been studied in [7, 15, 16]. The papers [15] and [16] study also the operators $H_{\mu}$ acting on distinct subspaces of the Bloch space, including $B M O A$, Besov spaces, and the $Q_{s}$-spaces.

In this paper we shall study the operators $H_{\mu}$ acting on mean Lipschitz spaces of analytic functions. 
If $f \in \mathcal{H o l}(\mathbb{D})$ has a non-tangential limit $f\left(e^{i \theta}\right)$ at almost every $e^{i \theta} \in \partial \mathbb{D}$ and $\delta>0$, we define

$$
\begin{aligned}
\omega_{p}(\delta, f) & =\sup _{0<|t| \leq \delta}\left(\frac{1}{2 \pi} \int_{-\pi}^{\pi}\left|f\left(e^{i(\theta+t)}\right)-f\left(e^{i \theta}\right)\right|^{p} d \theta\right)^{1 / p}, \quad \text { if } 1 \leq p<\infty \\
\omega_{\infty}(\delta, f) & =\sup _{0<|t| \leq \delta}\left(\operatorname{ess.~sup~}_{\theta \in[-\pi, \pi]}\left|f\left(e^{i(\theta+t)}\right)-f\left(e^{i \theta}\right)\right|\right) .
\end{aligned}
$$

Then $\omega_{p}(\cdot, f)$ is the integral modulus of continuity of order $p$ of the boundary values $f\left(e^{i \theta}\right)$ of $f$.

Given $1 \leq p \leq \infty$ and $0<\alpha \leq 1$, the mean Lipschitz space $\Lambda_{\alpha}^{p}$ consists of those functions $f \in \mathcal{H o l}(\mathbb{D})$ having a non-tangential limit almost everywhere for which $\omega_{p}(\delta, f)=O\left(\delta^{\alpha}\right)$, as $\delta \rightarrow 0$. If $p=\infty$ we write $\Lambda_{\alpha}$ instead of $\Lambda_{\alpha}^{\infty}$. This is the usual Lipschitz space of order $\alpha$.

A classical result of Hardy and Littlewood [17] (see also [9, Chapter 5]) asserts that for $1 \leq p \leq \infty$ and $0<\alpha \leq 1$, we have that $\Lambda_{\alpha}^{p} \subset H^{p}$ and

$$
\Lambda_{\alpha}^{p}=\left\{f \in \mathcal{H} \text { ol }(\mathbb{D}): M_{p}\left(r, f^{\prime}\right)=\mathrm{O}\left(\frac{1}{(1-r)^{1-\alpha}}\right)\right\} .
$$

It is known that if $1<p<\infty$ and $\alpha>\frac{1}{p}$ then each $f \in \Lambda_{\alpha}^{p}$ is bounded and has a continuous extension to the closed unit disc ([6], p.88). This is not true for $\alpha=\frac{1}{p}$, because the function $f(z)=\log (1-z)$ belongs to $\Lambda_{1 / p}^{p}$ for all $p \in(1, \infty)$. By a theorem of Hardy and Littlewood [9. Theorem 5.9] and of [6, Theorem 2.5] we have

$$
\Lambda_{1 / p}^{p} \subset \Lambda_{1 / q}^{q} \subset B M O A \quad 1 \leq p<q<\infty .
$$

The inclusion $\Lambda_{1 / p}^{p} \subset B M O A, 1 \leq p<\infty$ was proved to be sharp in a very strong sense in [3, 11, 12] using the following generalization of the spaces $\Lambda_{\alpha}^{p}$ which occurs frequently in the literature. Let $\omega:[0, \pi] \rightarrow[0, \infty)$ be a continuous and increasing function with $\omega(0)=0$ and $\omega(t)>0$ if $t>0$. Then, for $1 \leq p \leq \infty$, the mean Lipschitz space $\Lambda(p, \omega)$ consists of those functions $f \in H^{p}$ such that

$$
\omega_{p}(\delta, f)=O(\omega(\delta)), \quad \text { as } \delta \rightarrow 0 .
$$

With this notation we have $\Lambda_{\alpha}^{p}=\Lambda\left(p, \delta^{\alpha}\right)$.

The question of finding conditions on $\omega$ so that it is possible to obtain results on the spaces $\Lambda(p, \omega)$ analogous to those proved by Hardy and Littlewood for the spaces $\Lambda_{\alpha}^{p}$ has been studied by several authors (see [4, 5, 6]). We shall say that $\omega$ satisfies the Dini condition or that $\omega$ is a Dini-weight if there exists a positive constant $C$ such that

$$
\int_{0}^{\delta} \frac{\omega(t)}{t} d t \leq C \omega(\delta), \quad 0<\delta<1
$$

We shall say that $\omega$ satisfies the condition $b_{1}$ or that $\omega \in b_{1}$ if there exists a positive constant $C$ such that

$$
\int_{\delta}^{\pi} \frac{\omega(t)}{t^{2}} d t \leq C \frac{\omega(\delta)}{\delta}, \quad 0<\delta<1 .
$$

In order to simplify our notation, let $\mathcal{A} \mathcal{W}$ denote the family of all functions $\omega:[0, \pi] \rightarrow$ $[0, \infty)$ which satisfy the following conditions:

(i) $\omega$ is continuous and increasing in $[0, \pi]$.

(ii) $\omega(0)=0$ and $\omega(t)>0$ if $t>0$.

(iii) $\omega$ is a Dini-weight.

(iv) $\omega$ satisfies the condition $b_{1}$. 
The elements of $\mathcal{A W}$ will be called admissible weights. Characterizations and examples of admissible weights can be found in [4, 5].

Blasco and de Souza extended the above mentioned result of Hardy and Littlewood showing in [4, Th. 2.1] that if $\omega \in \mathcal{A} \mathcal{W}$ then,

$$
\Lambda(p, \omega)=\left\{f \text { analytic in } \mathbb{D}: M_{p}\left(r, f^{\prime}\right)=\mathrm{O}\left(\frac{\omega(1-r)}{1-r}\right) \text {, as } r \rightarrow 1\right\} .
$$

In [3, 11, 12] it is proved that if $1 \leq p<\infty$ and $\omega$ is an admissible weight such that

$$
\frac{\omega(\delta)}{\delta^{1 / p}} \rightarrow \infty, \text { as } \delta \rightarrow 0
$$

then there exists a function $f \in \Lambda(p, \omega)$ which is a not a normal function (see [1] for the definition). Since any Bloch function is normal, if follows that for such admissible weights $\omega$ one has that $\Lambda(p, \omega) \not \subset \mathcal{B}$.

One of the main results in [16] is the following one.

Theorem A ([16]). Let $\mu$ be a positive Borel measure on $[0,1)$ and let $X$ be a Banach space of analytic functions in $\mathbb{D}$ with $\Lambda_{1 / 2}^{2} \subset X \subset \mathcal{B}$. Then the following conditions are equivalent.

(i) The operator $\mathcal{H}_{\mu}$ is well defined in $X$ and, furthermore, it is a bounded operator from $X$ into he Bloch space $\mathcal{B}$.

(ii) The operator $\mathcal{H}_{\mu}$ is well defined in $X$ and, furthermore, it is a bounded operator from $X$ into $\Lambda_{1 / 2}^{2}$.

(iii) The measure $\mu$ is a 1-logarithmic 1-Carleson measure.

(iv) $\int_{[0,1)} t^{n} \log \frac{1}{1-t} d \mu(t)=\mathrm{O}\left(\frac{1}{n}\right)$.

A key ingredient in the proof of Theorem $\mathrm{A}$ is the fact that for any space $X$ with $\Lambda_{1 / 2}^{2} \subset$ $X \subset \mathcal{B}$ the functions $f \in X$ of the form $f(z)=\sum_{n=0}^{\infty} a_{n} z^{n}$ whose sequence of Taylor coefficients $\left\{a_{n}\right\}$ is a decreasing sequence of non-negative numbers are the same. Indeed, for such a function $f$ and such a space $X$ we have that $f \in X \Leftrightarrow a_{n}=\mathrm{O}\left(\frac{1}{n}\right)$. This result remains true if we substitute $\Lambda_{1 / 2}^{2}$ by $\Lambda_{1 / p}^{p}$ for any $p>1$. That is, the following result holds:

Lemma 1.1. Suppose that $1<p<\infty$ and let $f \in \mathcal{H}$ ol $(\mathbb{D})$ be of the form $f(z)=\sum_{n=0}^{\infty} a_{n} z^{n}$ with $\left\{a_{n}\right\}_{n=0}^{\infty}$ being a decreasing sequence of nonnegative numbers. If $X$ is a subspace of $\mathcal{H o l}(\mathbb{D})$ with $\Lambda_{1 / p}^{p} \subset X \subset \mathcal{B}$, then

$$
f \in X \quad \Leftrightarrow \quad a_{n}=\mathrm{O}\left(\frac{1}{n}\right) .
$$

Lemma 1.1 is a consequence of the following one which will be proved in Section2,

Lemma 1.2. Let $1<p<\infty, \omega \in \mathcal{A W}$ and let $f(z)=\sum_{n=0}^{\infty} a_{n} z^{n}$ with $\left\{a_{n}\right\}_{n=0}^{\infty}$ being a decreasing sequence of nonnegative numbers. Then

$$
f \in \Lambda(p, \omega) \quad \Leftrightarrow \quad a_{n}=\mathrm{O}\left(\frac{\omega(1 / n)}{n^{1-1 / p}}\right) .
$$

Using Lemma 1.1 and following the proof of Theorem $\mathrm{A}$ in [16], we obtain

Theorem 1.1. Suppose that $1<p<\infty$. Let $\mu$ be a positive Borel measure on $[0,1)$ and let $X$ be a Banach space of analytic functions in $\mathbb{D}$ with $\Lambda_{1 / p}^{p} \subset X \subset \mathcal{B}$. Then the following conditions are equivalent.

(i) The operator $\mathcal{H}_{\mu}$ is well defined in $X$ and, furthermore, it is a bounded operator from $X$ into he Bloch space $\mathcal{B}$. 
(ii) The operator $\mathcal{H}_{\mu}$ is well defined in $X$ and, furthermore, it is a bounded operator from $X$ into $\Lambda_{1 / p}^{p}$.

(iii) The measure $\mu$ is a 1-logarithmic 1-Carleson measure.

(iv) $\int_{[0,1)} t^{n} \log \frac{1}{1-t} d \mu(t)=\mathrm{O}\left(\frac{1}{n}\right)$.

As an immediate consequence of Theorem 1.1 we obtain the following result.

Corollary 1.1. Let $\mu$ be a positive Borel measure on $[0,1)$ and $1<p<\infty$. Then the operator $\mathcal{H}_{\mu}$ is well defined in $\Lambda_{1 / p}^{p}$ and, furthermore, it is a bounded operator from $\Lambda_{1 / p}^{p}$ into itself if and only if $\mu$ is a 1-logarithmic 1-Carleson measure.

Let us turn our attention now to the spaces $\Lambda(p, \omega)$ with $\frac{\omega(\delta)}{\delta^{1 / p}} \nearrow \infty, \delta \searrow 0$ which, as noted before, are not included in the Bloch space. We have the following result which shows that the situation is different from the one covered in Theorem 1.1 .

Theorem 1.2. Let $1<p<\infty, \omega \in \mathcal{A} \mathcal{W}$ with $\frac{\omega(\delta)}{\delta^{1 / p}} \nearrow \infty$ when $\delta \searrow 0$. The following conditions are equivalent:

(i) The operator $\mathcal{H}_{\mu}$ is well defined in $\Lambda(p, \omega)$ and, furthermore, it is a bounded operator from $\Lambda(p, \omega)$ into itself.

(ii) The measure $\mu$ is a Carleson measure.

The proofs of Lemma1.2 and Theorem 1.2 will be presented in Section 2. We close this section noticing that, as usual, we shall be using the convention that $C=C(p, \alpha, q, \beta, \ldots)$ will denote a positive constant which depends only upon the displayed parameters $p, \alpha, q, \beta \ldots$ (which sometimes will be omitted) but not necessarily the same at different occurrences. Moreover, for two real-valued functions $E_{1}, E_{2}$ we write $E_{1} \lesssim E_{2}$, or $E_{1} \gtrsim E_{2}$, if there exists a positive constant $C$ independent of the arguments such that $E_{1} \leq C E_{2}$, respectively $E_{1} \geq C E_{2}$. If we have $E_{1} \lesssim E_{2}$ and $E_{1} \gtrsim E_{2}$ simultaneously then we say that $E_{1}$ and $E_{2}$ are equivalent and we write $E_{1} \asymp E_{2}$.

\section{Proofs of the MAin RESUlts}

We start recalling that for a function $f(z)=\sum_{n=0}^{\infty} a_{n} z^{n}$ analytic in $\mathbb{D}$, the polynomials $\Delta_{j} f$ are defined as follows:

$$
\begin{gathered}
\Delta_{j} f(z)=\sum_{k=2^{j}}^{2^{j+1}-1} a_{k} z^{k}, \quad \text { for } j \geq 1, \\
\Delta_{0} f(z)=a_{0}+a_{1} z .
\end{gathered}
$$

The proof of Lemma[1.2] is based in the following result of Girela and González [14, Theorem 2].

Theorem B. Let $1<p<\infty$ and let $\omega$ be an admissible weight. If $f \in \mathcal{H}$ ol $(\mathbb{D})$ with $f(z)=\sum_{n=0}^{\infty} a_{n} z^{n}$ then

$$
f \in \Lambda(p, \omega) \Leftrightarrow\left\|\Delta_{N} f\right\|_{H^{p}}=O\left(\omega\left(\frac{1}{2^{N}}\right)\right) .
$$

Proof of Lemma 1.2. By Lemma A of [18], since $a_{n} \searrow 0$, we have

$$
\left\|\Delta_{N} f\right\|_{H^{p}} \asymp a_{2^{N}} 2^{N(1-1 / p)}, \quad N \geq 1 .
$$

So by Theorem B we have that

$$
f \in \Lambda(p, \omega) \Leftrightarrow a_{2^{N}} \lesssim \frac{\omega\left(1 / 2^{N}\right)}{2^{N(1-1 / p)}}, \quad N \geq 1
$$


This easily implies (1.2).

Lemma 2.1. Suppose that $1<p<\infty$. Let $\nu$ be a positive Borel measure on $[0,1)$, and let $\omega \in \mathcal{A W}$ satisfying that $x^{-1 / p} \omega(x) \nearrow \infty$, as $x \searrow 0$. Then following conditions are equivalent:

(i) $\nu_{n} \lesssim \frac{\omega(1 / n)}{n^{1-1 / p}}, n \geq 2$

(ii) $\nu([b, 1]) \lesssim(1-b)^{1-1 / p} \omega(1-b), b \in[0,1)$.

Proof. Suppose (i). Then we have that

$$
\begin{aligned}
1 & \gtrsim \frac{n^{1-1 / p} \nu_{n}}{\omega(1 / n)}=\frac{n^{1-1 / p}}{\omega(1 / n)} \int_{[0,1)} t^{n} d \nu(t) \geq \frac{n^{1-1 / p}}{\omega(1 / n)} \int_{[1-1 / n, 1)} t^{n} d \nu(t) \\
& \geq \frac{n^{1-1 / p}}{\omega(1 / n)} \nu([1-1 / n, 1))\left(1-\frac{1}{n}\right)^{n} \\
& \geq \frac{n^{1-1 / p}}{\omega(1 / n)} \nu([1-1 / n, 1)) \inf _{m \geq 2}\left(1-\frac{1}{m}\right)^{m} \\
& \gtrsim \frac{n^{1-1 / p}}{\omega(1 / n)} \nu([1-1 / n, 1)) .
\end{aligned}
$$

So $\nu([1-1 / n, 1)) \lesssim \frac{\omega(1 / n)}{n^{1-1 / p}}$ for $n \geq 2$.

Let now $b \in[1 / 2,1)$. There exists $n \geq 2$ such that $1-\frac{1}{n} \leq b<1-\frac{1}{n+1}$ so using the above we have that

$$
\nu([b, 1)) \leq \nu([1-1 / n, 1)) \lesssim \frac{\omega(1 / n)}{n^{1-1 / p}} .
$$

This, and the facts that $\omega(1 / n) n^{1 / p} \leq \omega(1 /(n+1))(n+1)^{1 / p}$ and that the weight $\omega$ increases give (ii).

Suppose now (ii). Then

$$
\begin{aligned}
\nu_{n} & =\int_{[0,1)} t^{n} d \nu(t)=n \int_{0}^{1} \nu([t, 1)) t^{n-1} d t \\
& \lesssim n \int_{0}^{1}(1-t)^{1-1 / p} \omega(1-t) t^{n-1} d t \\
& =n \int_{0}^{1-\frac{1}{n}}+\int_{1-\frac{1}{n}}^{1}\left((1-t)^{1-1 / p} \omega(1-t) t^{n-1} d t\right) .
\end{aligned}
$$

The first integral can be estimated bearing in mind that $(1-t)^{-1 / p} \omega(1-t) \nearrow \infty$ when $t \nearrow 1$ as follows

$$
\begin{aligned}
& n \int_{0}^{1-\frac{1}{n}}(1-t)^{1-1 / p} \omega(1-t) t^{n-1} d t \\
\leq & n^{1+1 / p} \omega(1 / n) \int_{0}^{1-\frac{1}{n}}(1-t) t^{n-1} d t \\
= & n^{1+1 / p} \omega(1 / n)\left(1-\frac{1}{n}\right)^{n}\left(\frac{1}{n}-\frac{n-1}{n(n+1)}\right) \\
\lesssim & \frac{\omega(1 / n)}{n^{1-1 / p}}
\end{aligned}
$$


To estimate of the second integral we use that $(1-t)^{1-1 / p} \omega(1-t) \searrow 0$ when $t \nearrow 1$ to obtain

$$
\begin{aligned}
& n \int_{1-\frac{1}{n}}^{1}(1-t)^{1-1 / p} \omega(1-t) t^{n-1} d t \\
\leq & n^{1 / p} \omega(1 / n) \int_{1-\frac{1}{n}}^{1} t^{n-1} d t \\
= & \frac{\omega(1 / n)}{n^{1-1 / p}}\left(1-\left(1-\frac{1}{n}\right)^{n}\right) \\
\lesssim & \frac{\omega(1 / n)}{n^{1-1 / p}}
\end{aligned}
$$

Then (i) follows.

Proof of Theorem 1.2. (i) $\Rightarrow$ (ii) Suppose that $\mathcal{H}_{\mu}: \Lambda(p, \omega) \rightarrow \Lambda(p, \omega)$ is bounded. By Lemma 1.2 we have that the function $f$ defined by $f(z)=\sum_{n=1}^{\infty} \frac{\omega(1 / n)}{n^{1-1 / p}} z^{n}$ belongs to the space $\Lambda(p, \omega)$ so, by the hypothesis, $\mathcal{H}_{\mu}(f)$ belongs also to $\Lambda(p, \omega)$. Now

$$
\mathcal{H}_{\mu}(f)(z)=\sum_{n=0}^{\infty}\left(\sum_{k=1}^{\infty} \frac{\omega(1 / k)}{k^{1-1 / p}} \mu_{n+k}\right) z^{n} .
$$

Notice that $\sum_{k=1}^{\infty} \frac{\omega(1 / k)}{k^{1-1 / p}} \mu_{n+k} \searrow 0, n \rightarrow \infty$, so using again Lemma 1.2 it holds that

$$
\sum_{k=1}^{\infty} \frac{\omega(1 / k)}{k^{1-1 / p}} \mu_{n+k}=\int_{[0,1)} t^{n} \sum_{k=1}^{\infty} \frac{\omega(1 / k)}{k^{1-1 / p}} t^{k} d \mu(t) \lesssim \frac{\omega(1 / n)}{n^{1-1 / p}}
$$

that is, the moments of the measure $\nu$ defined by

$$
d \nu(t)=\sum_{k=1}^{\infty} \frac{\omega(1 / k)}{k^{1-1 / p}} t^{k} d \mu(t)
$$

satisfy that

$$
\nu_{n} \lesssim \frac{\omega(1 / n)}{n^{1-1 / p}}
$$

so by Lemma 2.1 we have that $\nu([b, 1)) \lesssim(1-b)^{1-1 / p} \omega(1-b), b \in[0,1)$.

According to the definition of the measure

$$
\begin{aligned}
(1-b)^{1-1 / p} \omega(1-b) & \gtrsim \nu([b, 1))=\int_{[b, 1)} d \nu(t) \\
& =\int_{[b, 1)} \sum_{k=1}^{\infty} \frac{\omega(1 / k)}{k^{1-1 / p}} t^{k} d \mu(t) \\
& \geq \mu([b, 1)) \sum_{k=1}^{\infty} \frac{\omega(1 / k)}{k^{1-1 / p}} b^{k}
\end{aligned}
$$


and the sum can be estimated as follows

$$
\begin{aligned}
\sum_{k=1}^{\infty} \frac{\omega(1 / k)}{k^{1-1 / p}} b^{k} & \asymp \int_{1}^{\infty} \frac{\omega(1 / x)}{x^{1-1 / p}} b^{x} d x \\
& \geq \int_{1}^{\frac{1}{1-b}} \frac{\omega(1 / x)}{x^{1-1 / p}} b^{x} d x \\
& \geq(1-b)^{1-1 / p} \omega(1-b) b^{\frac{1}{1-b}}\left(\frac{1}{1-b}-1\right) \\
& \gtrsim \frac{\omega(1-b)}{(1-b)^{1 / p}} .
\end{aligned}
$$

Finally, putting all together we have that

$$
\mu([b, 1)) \lesssim 1-b
$$

so $\mu$ is a Carleson measure.

(ii) $\Rightarrow$ (i) To prove this implication we need to use the integral operator $I_{\mu}$ considered in [7, 10, 15, 16] which is closely related to the operator $\mathcal{H}_{\mu}$.

If $\mu$ is a positive Borel measure on $[0,1)$ and $f \in \mathcal{H o l}(\mathbb{D})$, we shall write throughout the paper

$$
I_{\mu}(f)(z)=\int_{[0,1)} \frac{f(t)}{1-t z} d \mu(t)
$$

whenever the right hand side makes sense and defines an analytic function in $\mathbb{D}$. It turns out that the operators $H_{\mu}$ and $I_{\mu}$ are closely related. Indeed, as shown in the just mentioned papers, it turns out that if $f$ is good enough $H_{\mu}(f)$ and $I_{\mu}(f)$ are well defined and coincide.

Suppose that $\mu$ is a Carleson measure supported on $[0,1)$ and let $f \in \Lambda(p, \omega)$. We claim that

$$
\int_{[0,1)} \frac{|f(t)|}{|1-t z|} d \mu(t)<\infty
$$

Indeed, using Lemma 3 of [14] we have that

$$
f \in \Lambda(p, \omega) \Rightarrow|f(z)| \lesssim \frac{\omega(1-|z|)}{(1-|z|)^{1 / p}}, \quad z \in \mathbb{D}
$$

Then we obtain

$$
\begin{aligned}
\int_{[0,1)} \frac{|f(t)|}{|1-t z|} d \mu(t) & \leq \frac{1}{1-|z|} \int_{[0,1)}|f(t)| d \mu(t) \\
& \lesssim \frac{1}{1-|z|} \int_{[0,1)} \frac{\omega(1-t)}{(1-t)^{1 / p}} d \mu(t) .
\end{aligned}
$$

If we choose $r \in[0,1)$ we can split the integral in the intervals $[0, r)$ and $[r, 1)$. In the first one, as $\omega$ is an increasing weight we have

$$
\begin{aligned}
\int_{[0, r)} \frac{\omega(1-t)}{(1-t)^{1 / p}} d \mu(t) & \leq \omega(1) \int_{[0, r)} \frac{d \mu(t)}{(1-t)^{1 / p}} \\
& \leq \omega(1) \int_{[0,1)} \frac{d \mu(t)}{(1-t)^{1 / p}} \\
& \lesssim 1,
\end{aligned}
$$


because $\mu$ is a Carleson measure. Using this and the condition $\frac{\omega(\delta)}{\delta^{1 / p}} \nearrow \infty$, as $\delta \searrow 0$ we can estimate the other integral as follows

$$
\begin{aligned}
\int_{[r, 1)} \frac{\omega(1-t)}{(1-t)^{1 / p}} d \mu(t) & \leq \frac{\omega(1-r)}{(1-r)^{1 / p}} \int_{[r, 1)} d \mu(t) \\
& \lesssim \omega(1-r)(1-r)^{1-1 / p} \\
& \lesssim 1
\end{aligned}
$$

So we have that for $f \in \Lambda(p, \omega)$ and $z \in \mathbb{D}$, (2.1) holds. This implies that $I_{\mu}(f)$ is well defined, and, using Fubini's theorem and standard arguments it follows easily that $\mathcal{H}_{\mu}(f)$ is also well defined and that, furthermore,

$$
\mathcal{H}_{\mu}(f)(z)=I_{\mu}(f)(z), \quad z \in \mathbb{D} .
$$

Now we have,

$$
I_{\mu}(f)^{\prime}(z)=\int_{[0,1)} \frac{t f(t)}{(1-t z)^{2}} d \mu(t), \quad z \in \mathbb{D},
$$

so the mean of order $p$ of $I_{\mu}(f)^{\prime}$ has the form

$$
M_{p}\left(r, I_{\mu}(f)^{\prime}\right)=\left(\frac{1}{2 \pi} \int_{-\pi}^{\pi}\left|\int_{[0,1)} \frac{t f(t)}{\left(1-t r e^{i \theta}\right)^{2}} d \mu(t)\right|^{p} d \theta\right)^{1 / p} .
$$

Using again (2.2), the Minkowski inequality and a classical estimation of integrals we obtain that

$$
\begin{aligned}
M_{p}\left(r, I_{\mu}(f)^{\prime}\right) & \lesssim \int_{[0,1)}|f(t)|\left(\int_{-\pi}^{\pi} \frac{d \theta}{\left|1-t r e^{i \theta}\right|^{2 p}}\right)^{1 / p} d \mu(t) \\
& \lesssim \int_{[0,1)} \frac{|f(t)|}{(1-t r)^{2-1 / p}} d \mu(t) \\
& \lesssim \int_{[0,1)} \frac{\omega(1-t)}{(1-t)^{1 / p}(1-t r)^{2-1 / p}} d \mu(t) .
\end{aligned}
$$

At this point we split the integrals on the sets $[0, r)$ and $[r, 1)$.

In the first integral we use that $x^{-1 / p} \omega(x) \nearrow \infty$, as $x \searrow 0$, and the fact that if $\mu$ is a Carleson measure (so that $\mu_{n}=\int_{[0,1)} t^{n} d \mu(t) \lesssim \frac{1}{n}$ ) to obtain

$$
\begin{aligned}
\int_{[0, r)} \frac{\omega(1-t)}{(1-t)^{1 / p}(1-t r)^{2-1 / p}} d \mu(t) & \leq \frac{\omega(1-r)}{(1-r)^{1 / p}} \int_{[0, r)} \frac{d \mu(t)}{(1-t r)^{2-1 / p}} \\
& \leq \frac{\omega(1-r)}{(1-r)^{1 / p}} \int_{[0,1)} \frac{d \mu(t)}{(1-t r)^{2-1 / p}} \\
& \lesssim \frac{\omega(1-r)}{(1-r)^{1 / p}} \sum_{n=1}^{\infty} n^{1-1 / p} r^{n} \int_{[0,1)} t^{n} d \mu(t) \\
& \lesssim \frac{\omega(1-r)}{(1-r)^{1 / p}} \sum_{n=1}^{\infty} \frac{r^{n}}{n^{1 / p}} \\
& \lesssim \frac{\omega(1-r)}{(1-r)}
\end{aligned}
$$

In the second integral we use that $\omega$ is an increasing weight and the fact that the measure $\mu$ being a Carleson measure is equivalent to saying that the measure $\nu$ defined by $d \nu(t)=$ 
$\frac{d \mu(t)}{(1-t)^{1 / p}}$ is a $1-\frac{1}{p}$-Carleson measure so that the moments $\nu_{n}$ of $\nu$ satisfy $\nu_{n} \lesssim \frac{1}{n^{1-\frac{1}{p}}}$. Then we obtain

$$
\begin{aligned}
\int_{[r, 1)} \frac{\omega(1-t)}{(1-t)^{1 / p}(1-t r)^{2-1 / p}} d \mu(t) & \leq \omega(1-r) \int_{[r, 1)} \frac{d \nu(t)}{(1-t r)^{2-1 / p}} \\
& \leq \omega(1-r) \int_{[0,1)} \frac{d \nu(t)}{(1-t r)^{2-1 / p}} \\
& \lesssim \omega(1-r) \sum_{n=1}^{\infty} n^{1-1 / p} r^{n} \int_{[0,1)} t^{n} d \nu(t) \\
& \lesssim \omega(1-r) \sum_{n=1}^{\infty} r^{n} \\
& =\frac{\omega(1-r)}{(1-r)}
\end{aligned}
$$

Therefore $I_{\mu}(f) \in \Lambda(p, \omega)$ and then the operator $I_{\mu}$ (and hence the operator $\mathcal{H}_{\mu}$ ) is bounded from $\Lambda(p, \omega)$ into itself.

\section{REFERENCES}

[1] J. M. Anderson, J. Clunie and Ch. Pommerenke, On Bloch functions and normal functions, J. Reine Angew. Math. 270 (1974), 12-37.

[2] G. Bao and H. Wulan, Hankel matrices acting on Dirichlet spaces, J. Math. Anal. Appl. 409 (2014), no. 1, 228-235.

[3] O. Blasco, D. Girela and M. A. Márquez, Mean growth of the derivative of analytic functions, bounded mean oscillation and normal functions, Indiana Univ. Math. J. 47 (1998), no. 2, 893-912.

[4] O. Blasco and G. S. de Souza, Spaces of analytic functions on the disc where the growth of $M_{p}(F, r)$ depends on a weight, J. Math. Anal. Appl. 147 (1989), no. 2, 580-598.

[5] S. Bloom and G. S. de Souza, Weighted Lipschitz spaces and their analytic characterizations, Constr. Approx. 10 (1994), 339-376.

[6] P. Bourdon, J. Shapiro and W. Sledd, Fourier series, mean Lipschitz spaces and bounded mean oscillation, Analysis at Urbana 1, Proc. of the Special Yr. in Modern Anal. at the Univ. of Illinois 1986-87, (E.R. Berkson, N.T. Peck and J. Uhl, eds.), London Math. Soc. Lecture Notes Ser. 137, Cambridge Univ. Press, (1989), 81-110.

[7] Ch. Chatzifountas, D. Girela and J. A. Peláez, A generalized Hilbert matrix acting on Hardy spaces, J. Math. Anal. Appl. 413 (2014), no. 1, 154-168.

[8] E. Diamantopoulos and A. G. Siskakis, Composition operators and the Hilbert matrix, Studia Math. 140 (2000), 191-198.

[9] P. L. Duren, Theory of $H^{p}$ Spaces, Academic Press, New York-London 1970. Reprint: Dover, Mineola, New York 2000.

[10] P. Galanopoulos and J. A. Peláez, A Hankel matrix acting on Hardy and Bergman spaces, Studia Math. 200, 3, (2010), 201-220.

[11] D. Girela, On a theorem of Privalov and normal functions, Proc. Amer. Math. Soc. 125 (1997), 433-442.

[12] D. Girela, Mean Lipschitz spaces and bounded mean oscillation, Illinois J. Math. 41 (1997), 214-230.

[13] D. Girela, Analytic functions of bounded mean oscillation. In: Complex Function Spaces, Mekrijärvi 1999 Editor: R. Aulaskari. Univ. Joensuu Dept. Math. Rep. Ser. 4, Univ. Joensuu, Joensuu (2001) pp. 61-170.

[14] D. Girela and C. González, Some results on mean Lipschitz spaces of analytic functions, Rocky Mountain J. Math. 30 (2000), no. 3, 901-922.

[15] D. Girela and N. Merchán, A generalized Hilbert operator acting on conformally invariant spaces, to appear in Banach. J. Math. Anal. (available at https://arxiv.org/pdf/1612.08304.pdf)

[16] D. Girela and N. Merchán, A Hankel matrix acting on spaces of analytic functions, preprint (available at https://arxiv.org/pdf/1706.04079.pdf)

[17] G.H . Hardy and J. E. Littlewood, Some properties of fractional integrals, II, Math. Z. 34 (1932), 403-439. 
[18] M. Pavlović, Analytic functions with decreasing coefficients and Hardy and Bloch spaces, Proc. Edinburgh Math. Soc. Ser. 2 56, 2, (2013), 623-635.

[19] V. Peller, Hankel operators and their applications, Springer Monographs in Mathematics, Springer-Verlag, New York, 2003.

[20] S. C. Power, Vanishing Carleson measures, Bull. London Math. Soc. 12, (1980), 207-210.

[21] H. Widom, Hankel matrices, Trans. Amer. Math. Soc. 121, (1966), 1-35.

[22] R. Zhao, On logarithmic Carleson measures, Acta Sci. Math. (Szeged) 69 (2003), no. 3-4, 605-618.

[23] K. Zhu, Operator Theory in Function Spaces, Second Edition, Math. Surveys and Monographs, 138 (2007).

Análisis Matemático, Facultad de Ciencias, Universidad de Málaga, 29071 Málaga, Spain

E-mail address: noel@uma.es 